\title{
Why could ice ages be unpredictable?
}

\section{Crucifix}

Earth and Life Institute, Georges Lemaitre Centre for Earth and Climate Research Université catholique de Louvain, Louvain-la-Neuve, Belgium

Correspondence to: M. Crucifix (michel.crucifix@uclouvain.be)

Received: 4 February 2013 - Published in Clim. Past Discuss.: 20 February 2013

Revised: 30 June 2013 - Accepted: 28 August 2013 - Published: 9 October 2013

\begin{abstract}
It is commonly accepted that the variations of Earth's orbit and obliquity control the timing of Pleistocene glacial-interglacial cycles. Evidence comes from power spectrum analysis of palaeoclimate records and from inspection of the timing of glacial and deglacial transitions. However, we do not know how tight this control is. Is it, for example, conceivable that random climatic fluctuations could cause a delay in deglaciation, bad enough to skip a full precession or obliquity cycle and subsequently modify the sequence of ice ages?

To address this question, seven previously published conceptual models of ice ages are analysed by reference to the notion of generalised synchronisation. Insight is being gained by comparing the effects of the astronomical forcing with idealised forcings composed of only one or two periodic components. In general, the richness of the astronomical forcing allows for synchronisation over a wider range of parameters, compared to periodic forcing. Hence, glacial cycles may conceivably have remained paced by the astronomical forcing throughout the Pleistocene.

However, all the models examined here show regimes of strong structural dependence on parameters. This means that small variations in parameters or random fluctuations may cause significant shifts in the succession of ice ages. Whether the actual system actually resides in such a regime depends on the amplitude of the effects associated with the astronomical forcing, which significantly differ across the different models studied here. The possibility of synchronisation on eccentricity is also discussed and it is shown that a high Rayleigh number on eccentricity, as recently found in observations, is no guarantee of reliable synchronisation.
\end{abstract}

\section{Introduction}

Hays et al. (1976) showed that Southern Ocean climate benthic records exhibit spectral peaks around 19, 23-24, 42 and 100 thousand years (thousand years are henceforth denoted "ka"). More or less concomitantly Berger (1977) showed, based on celestial mechanics, that the power spectrum of climatic precession was dominated by periods of 19, 22 and $24 \mathrm{ka}$, and that of obliquity was dominated by a period of $41 \mathrm{ka}$. These authors concluded that the succession of ice ages is somehow controlled by the astronomical forcing.

However, experiments with numerical models have also suggested that the precise timing of the glaciation or deglaciation could sensitively depend on parameters that are not well known. Weertman (1976), for example, showed that the natural course of the ice volume from the present-day (ignoring anthropogenic forcing) could either be glacial inception or a long interglacial, depending on whether a certain parameter is set to 2.75 or 2.745 . Paillard (2001) observed similar phenomena using a model published in Paillard (1998).

Shown in Fig. 1, for instance, are two further examples of ice volume history reproduced with models previously published in the palaeoclimate modelling literature (Saltzman and Maasch, 1990; Tziperman et al., 2006). In both cases, small changes in model parameters do, at some stage in the climate history, induce a shift in the sequence of ice ages.

The question of the stability of the ice age sequence is not new. As early as 1980, Imbrie and Imbrie (1980) wondered whether "nonorbitally forced high-frequency fluctuations may have caused the system to flip or flop in an unpredictable fashion." They also noted that "the regularity of the 100-ka cycle, and particularly its phase coherence with the 100-ka eccentricity cycle, argue for predictability". The importance of eccentricity as a control of ice ages was recently 
Forcing (mid-June insolation at $65 \mathrm{~N}$ )
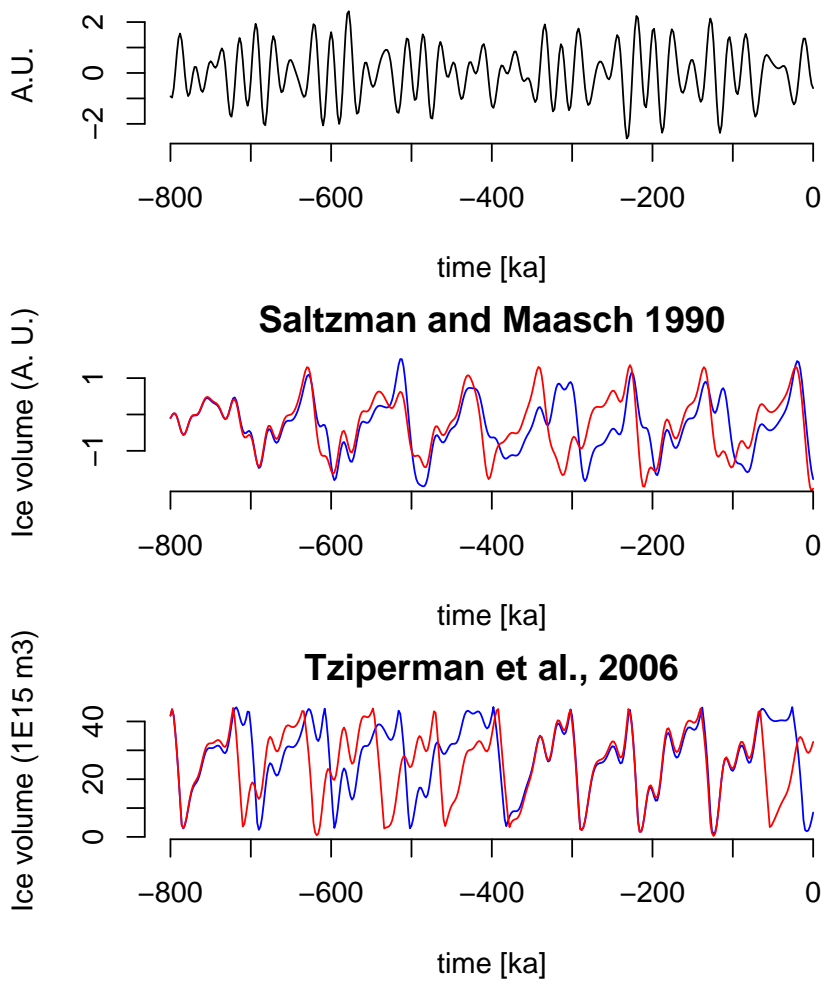

Fig. 1. Ice volume simulated with two models previously published: Saltzman and Maasch (1990) and Tziperman et al. (2006), forced by normalised insolation at $65^{\circ} \mathrm{N}$. The blue lines are obtained with the published parameters; the latter were slightly changed to obtain the red ones: $p_{0}=0.262 \mathrm{~Sv}$ instead of $0.260 \mathrm{~Sv}$ in Tziperman et al. (2006), and $w=0.6$ instead of $w=0.5$ in Saltzman and Maasch (1990). While the qualitative aspect of the curves are preserved, the timing of ice ages is affected by the parameter changes.

brought up by Lisiecki (2010) and Rial (2004); Rial et al. (2013).

Tziperman et al. (2006) already observed that a conceptual model fitting the ice age curve is no proof that the correct physical mechanisms have been identified, but is rather an indication of the powerful role of the astronomical forcing as a pacemaker. Our purpose is to take this approach one step further by showing that the powerful action of the astronomical forcing as a pacemaker does not guarantee the stability of the ice age sequence.

A first step was proposed in De Saedeleer et al. (2013), where a highly idealised model of ice ages (in essence, a van der Pol oscillator) displays a wide regime of synchronisation when forced to the astronomical forcing. Inspection of the time-averaged largest Lyapunov exponents, as well as basins of attractions, allowed us to conclude that that the forced system goes through times of unreliable synchronisation, so that the succession of ice ages may sensitively depend on fluctuations added to the system.
This work is further developed here in three directions: the role of separate and combined influences of different components of the astronomical forcing is being analysed more systematically. This allows us to relate the instabilities noted in palaeoclimate models to the literature on quasi-periodic forcings with two components only, and more specifically to the concept of strange non-chaotic attractor. The possibility of synchronisation on eccentricity is also briefly discussed. Second, the effects of additive fluctuations and those of parameter changes are being related qualitatively. Finally, the analysis is applied to six other palaeoclimate models, which provides a better basis to estimate the robustness of the conclusions.

Providing no conclusions as to whether or not glacialinterglacial cycles are indeed predictable, the present work focuses entirely on the possibility of dynamical stability of simple ice age models. Making conclusions about the real world requires first an elaborate account of the effects associated with model discrepancy (which is stochastic) that need to be quantified through a process of statistical inference.

\section{The van der Pol oscillator}

\subsection{Model definition}

Before going further a word of justification is needed about the use of the van der Pol oscillator as the starting point of this study.

The succession of glaciations and terminations is commonly understood as a phenomenon of relaxation, during which regimes of linear response to the astronomical forcing alternate with non-linear phenomena during which the attracting point of the system changes.

The idea, popularised by Paillard (1998), is already implicit in the works of Oerlemans (1980), based on experiments with an ice sheet-lithosphere model. However, the understanding of the nature of the instabilities that are necessary to cause the succession of glacial-interglacial cycles has considerably evolved over time, as it may involve the lithosphere (Oerlemans, 1980), the ocean biogeochemistry (Saltzman et al., 1984), a combination of both (Saltzman and Verbitsky, 1993), the ocean circulation (Paillard and Parrenin, 2004) or sea-ice-atmospheric interactions (Gildor and Tziperman, 2000).

The van der Pol model is thus chosen here as it is one of the simplest relaxation oscillators, without consideration of the exact physical mechanism that causes the ice age oscillations.

It is a dynamical system of two ordinary differential equations:

$$
\begin{aligned}
& \frac{\mathrm{d} x}{\mathrm{~d} t}=-\frac{1}{\tau}(F(t)+\beta+y) . \\
& \frac{\mathrm{d} y}{\mathrm{~d} t}=\frac{\alpha}{\tau}\left(y-y^{3} / 3+x\right),
\end{aligned}
$$


with $(\boldsymbol{x}, \boldsymbol{y})$ as the climate state vector, $\tau$ a time constant, $\alpha$ a time-decoupling factor, $\beta$ a bifurcation parameter and $F(t)$ the forcing. The parameter $\beta$ does not appear in the original van der Pol equations (van der Pol, 1926), and the present variant is sometimes referred to as the biased van der Pol model.

The autonomous (i.e. $F(t)=0$ ) model displays selfsustained oscillations as long as $|\beta|<1$. For later reference the period of the unforced oscillator is denoted $T_{\mathrm{n}}(\tau)$. The variable $x$ then follows a saw-tooth periodic cycle, the asymmetry of which is controlled by $\beta$.

We chose $\alpha=30$. This choice implies that variable $y$ undergoes rapid variations compared to $x$, and it may therefore be referred to as the "fast" variable. In climate terms, $x$ may be interpreted as a glaciation index, which slowly accumulates the effects of the astronomical forcing $F(t)$, while $y$, which shifts between approximately -1 and 1 , might be interpreted as some representation of the ocean or carbon cycle dynamics.

Again, the van der Pol oscillator is only taken here as a starting point, its representativeness of the physics of ice ages being admittedly contentious. Models with more explicit interpretations of ice age physics will be discussed in Sect. 3 .

In ice age models the forcing function is generally one or several insolation curves, computed for specific seasons and latitudes. The rationale behind this choice is that whichever insolation is used it is, to a very good approximation, a linear combination of climatic precession and obliquity (Loutre, 1993, see also Appendix A). The choice of one specific insolation curve may be viewed as a modelling decision about the effective forcing phase of climatic precession, and the relative amplitudes of the forcings due to precession and obliquity. In turn, climatic precession and obliquity can be expressed as a sum of sines and cosines of various amplitudes and frequencies (Berger, 1978), so that $F(t)$ can be modelled as a linear combination of about a dozen of dominant periodic signals, plus a series of smaller amplitude components. They are shown in Fig. 2. More details are given in Sect. 2.4. With these hypotheses the van der Pol model may be tuned so that the benthic curve over the last $800000 \mathrm{yr}$ is reasonably well reproduced (Fig. 3).

Abundant literature analyses the response of the van der Pol oscillator to a periodic forcing (e.g. Mettin et al., 1993; Guckenheimer et al., 2003, and references therein). The response of oscillators to the sum of two periodic forcings has been the focus of attention because it leads to the emergence of "strange non-chaotic attractors", a concept first introduced by Grebogi et al. (1984) and further studied in, among others, Wiggins (1987); Romeiras and Ott (1987); Kapitaniak and Wojewoda (1990, 1993); Belogortsev (1992); Feudel et al. (1997); and Glendinning et al. (2000). To our knowledge, however, there is no systematic study of the response of an oscillator to a signal of the form of the astronomical forcing. Le Treut and Ghil (1983), for example, represented the astronomical forcing as a sum of only two or three periodic
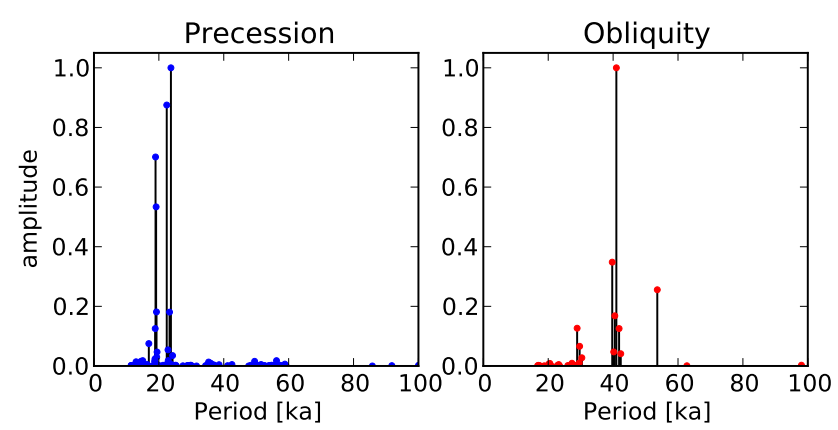

Fig. 2. Spectral decomposition of precession and obliquity given by Berger (1978), scaled here such that the strongest components have amplitude 1.

components and it will be shown here that it is worth considering the astronomical forcing with all its complexity.

\subsection{Periodic forcing}

Consider a sine-wave forcing $\left(F(t)=\gamma \sin \left(2 \pi / P_{1} t+\phi_{P 1}\right)\right)$, with period $P_{1}=23716 \mathrm{yr}$ and $\phi_{P 1}=32.01^{\circ}$. This is the first component of the harmonic development of climatic precession (Berger, 1978). If certain conditions are met - they will soon be given - the van der Pol oscillator may become synchronised on the forcing. Synchronised means, in this particular context, that the response of the system displays $p$ cycles within $q$ forcing periods, where $p$ and $q$ are integers. It is said that the system is in a $p: q$ synchronisation regime (Pikovski et al., 2001, p. 66-67). The output is periodic, and its period is equal to $q \times P_{1}$.

There are several ways to identify the synchronisation in the output of a dynamical system. One method is to plot the state of the system at a given time $t$, and then superimpose on that plot the state of the system at every time $t+n P_{1}$, where $n$ is integer. The system is synchronised if only $q$ distinct points appear on the graph, discarding transient effects associated with initial conditions. These correspond to the stable fixed points of the iteration bringing the system from $t$ to $t+q P_{1}$. In the following we refer to this kind of plot as a "stroboscopic section" of period $P_{1}$ (Fig. $4 \mathrm{a}$ ). If the system is not synchronised, then there are two options: the stroboscopic section is a closed curve (the response is quasi-periodic), or a figure with a strange geometry (the response is a-periodic).

There is another, equivalent way to identify synchronisation. Suppose that the system is started from arbitrary initial conditions. Then, plot the system state at a given time $t$, long enough after the initial conditions. Repeat the experiment with another set of initial conditions, superimpose the result on the plot, and so on with a very large number of different initial conditions. In doing so one constructs the section of the global pullback attractor at time $t$ (henceforth referred to as the pullback section); the pullback attractor itself being the continuation of this figure over all times (Fig. $4 \mathrm{~b}$ ). 
van der Pol oscillator forced by astronomical forcing

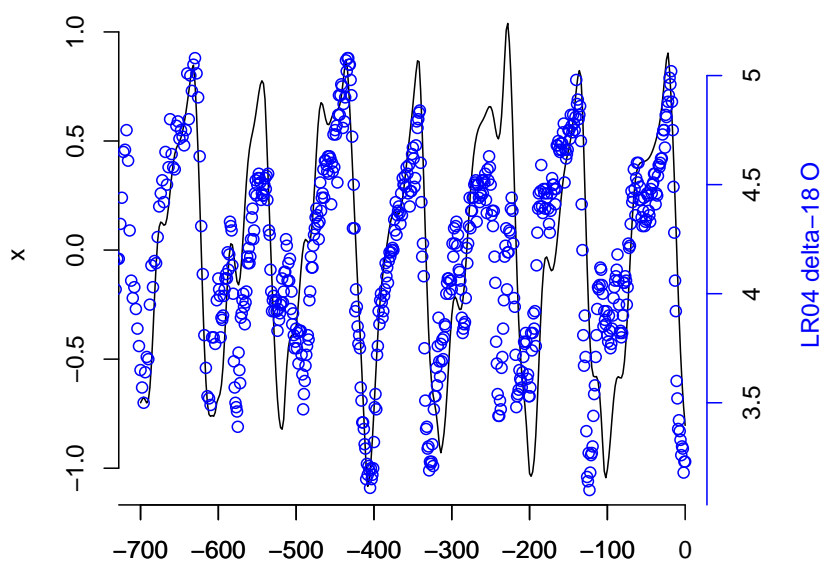

Time [ka]

Fig. 3. Simulation with the van der Pol oscillator (Eqs. 1-4), forced by astronomical forcing and compared with the benthic record of Lisiecki and Raymo (2005). Parameters are: $\alpha=30, \beta=0.75$, $\gamma_{p}=\gamma_{o}=0.6, \tau=36.2 \mathrm{ka}$.

Each component of the global pullback attractor (two are illustrated in Fig. 4b) is a local pullback attractor. Rasmussen (2000, Chap. 2) reviews all the relevant mathematical formalism.

In the particular case of a periodic forcing, the stroboscopic section and the pullback section are often identical (Fig. 5) ${ }^{1}$.

The number of points on the pullback section may then be estimated for different combinations of parameters and we can use this as a criteria to detect synchronisation. This is done in Fig. 6 for a range of $\gamma$ and $\tau$. It turns out that synchronisation regimes are organised in the form of triangles, known in the dynamical system literature as Arnol'd tongues (Pikovski et al., 2001, p. 52). A $p: q$ synchronisation regime appears when the ratio between the natural period $\left(T_{\mathrm{n}}\right)$ and the forcing period is close to $q / p$. The tolerance, i.e. how distant this ratio can afford to be with respect to $q / p$, increases with the forcing amplitude and decreases with $p$ and $q$. Synchronisation is weakest (least reliable) near the edge of the tongues. Unreliable synchronisation characterises a system that is synchronised, but in which small fluctuations may

\footnotetext{
${ }^{1}$ This property derives from the system invariance with respect a time translation by $P$. There will be, however, cases where different initial conditions will create different stroboscopic plots. For example two $1: 2$ synchronisation regimes co-exist in the forced van der Pol oscillator, so that there are four distinct local pullback attractors, while only two points will appear on a stroboscopic plot started from a single set of initial conditions. Rigorously, the global pullback attractor at time $t$ is identical to the global attractor of the iteration $t+n P$, and the global pullback sections at two times $t$ and $t^{\prime}$ are homeomorphic.
}

(a)

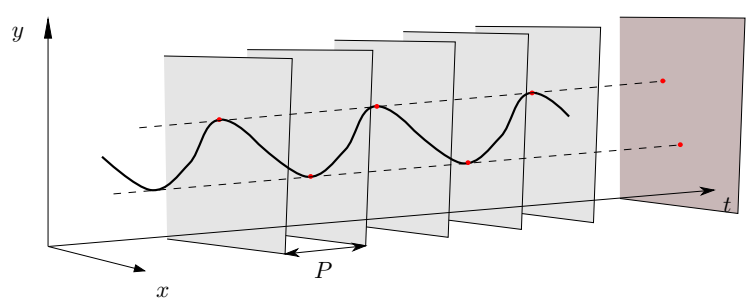

(b)

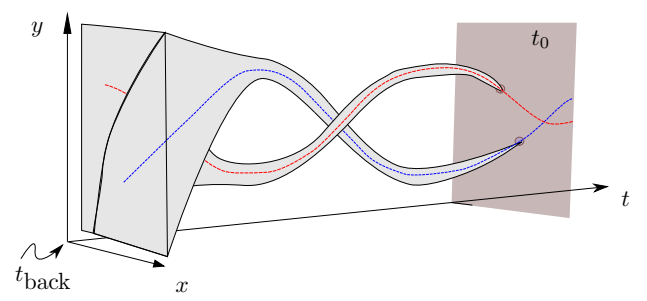

Fig. 4. (a) The stroboscopic section is obtained by superimposing the system state every forcing period $(P)$, here illustrated for a $2: 1$ synchronisation. (b) The pullback section at time $t_{0}$ is obtained by superimposing the system states obtained by initialising the system with the ensemble of all possible initial conditions far back in time, here at $t_{\text {back }}$. The particular example shows a global pullback attractor made of two local pullback attractors (in dashed red and blue), the sections of which are seen at $t$. Also shown is the convergence of initial conditions towards the pullback attractors, in grey.

cause episodes of desynchronisation. In the particular case of periodic forcing the episode of desynchronisation is called a phase slip, as is well explained in Pikovski et al. (2001, p. 54)

Using the pullback attractor to identify synchronisation is not a very efficient method in the periodic forcing case. Arclength continuation methods are faster and more accurate (e.g. Schilder and Peckham, 2007, and references therein). It is shown, however, in De Saedeleer et al. (2013) that the pullback section method gives results that are acceptable enough for our purpose, and it is adopted here because it provides a more intuitive starting point to characterise synchronisation with multi-periodic forcings.

\subsection{Synchronisation on two periods}

Consider now a forcing function that is the sum of two periodic signals. Two cases are considered here: the two forcing periods differ by a factor of about 2 , and the two forcing periods are close.

\subsection{1 $P_{1}=23716 \mathrm{ka}$ and $O_{1}=41000 \mathrm{ka}$}

We adopt $\quad F(t)=\gamma\left[\sin \left(2 \pi / P_{1} t+\phi_{P 1}\right)+\cos \left(2 \pi / O_{1} t+\right.\right.$ $\left.\left.\phi_{O 1}\right)\right]$, with $P_{1}=23716 \mathrm{ka}, \quad O_{1}=41000 \mathrm{ka}$ and $\phi_{P 1}=32.01^{\circ}$ and $\phi_{O 1}=251.09^{\circ} . P_{1}$ is the first period in the development of precession, $O_{1}$ is the first period in the development of obliquity and $\phi_{P 1}, \phi_{O 1}$ the corresponding phases given by Berger (1978), so that $F(t)$ may already be 

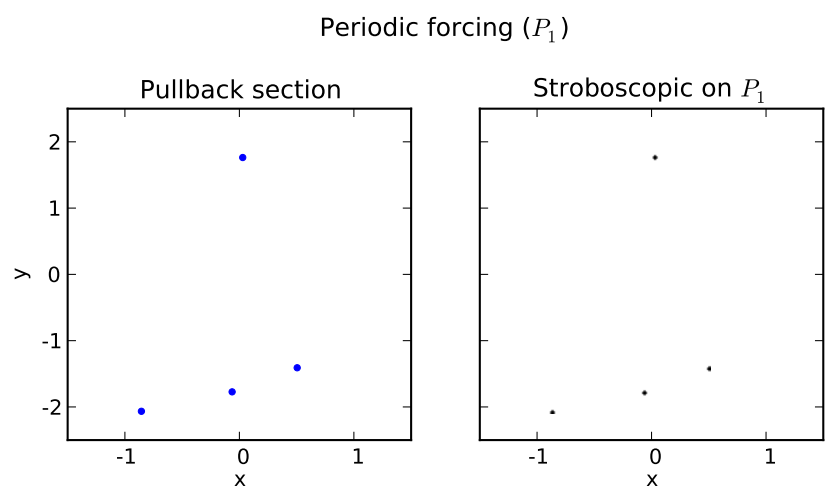

Fig. 5. Pullback (at $\left.t_{0}=0\right)$ and stroboscopic sections $\left(t=0+n P_{1}\right)$ obtained with the van der Pol oscillator, with parameters $\alpha=$ $30, \beta=0.7, \tau=36$ forced by $F(t)=\gamma \sin \left(2 \pi / P_{1} t+\phi_{P 1}\right), P_{1}=$ $23.7 \mathrm{ka}, \phi_{P 1}=32.01^{\circ}$ and $\gamma=0.6$. The two plots indicate a case of $4: 1$ synchronisation, and they are identical because the forcing is periodic.

viewed as a very rough representation of the astronomical forcing.

Let us begin with $\tau=36 \mathrm{ka}$, which corresponds to a limit cycle in the van der Pol oscillator of period $T_{\mathrm{n}}=98.79 \mathrm{ka}$, and consider the stroboscopic section on $P_{1}$ (Fig. 7, line 1). Forcing amplitude $\gamma$ is set to 0.6. Due to the presence of the $O_{1}$ forcing, the four points of the periodic case seen in Fig. 5 have mutated into four local attractors, which appear as closed curves (some are very flat). Every time $P_{1}$ elapses, the system visits a different local attractor. They are attractors in the sense that they attract solutions of the iteration, bringing the system from $t$ to $t+4 \cdot P_{1}$. In this particular example, the system is said to be phase- or frequency-locked on $P_{1}$ with ratio $1: 4$ (Pikovski et al., 2001, p. 68), because on average, one ice age cycle takes four precession cycles, even though the response is no longer periodic. In this example, the curves on the $P_{1}$ stroboscopic section nearly touch each other. This implies that synchronisation is not reliable since a solution captured by one of these attractors could easily escape and fall into the basin of attraction of another local attractor. One can also see that it is not synchronised on $O_{1}$ since the stroboscopic section of period $O_{1}$ shows one closed curve englobing all possible phases. It may also be said that the system is synchronised in the generalised sense (Pikovski et al., 2001, p. 150), because the pullback section is made of only four points: starting from arbitrary conditions, the system converges to only a small number of solutions at any time $t$. It is also stable in the Lyapunov sense, a point that will not be further discussed here, but see De Saedeleer et al. (2013).

Consider now $\tau=41 \mathrm{ka}$. The four closed curves on the P1-stroboscopic section have collided and merged into one attractor with strange geometry. A similar figure appears on the E1-stroboscopic section. The phenomenon of strange non-chaotic attractor has been described by Grebogi et al.

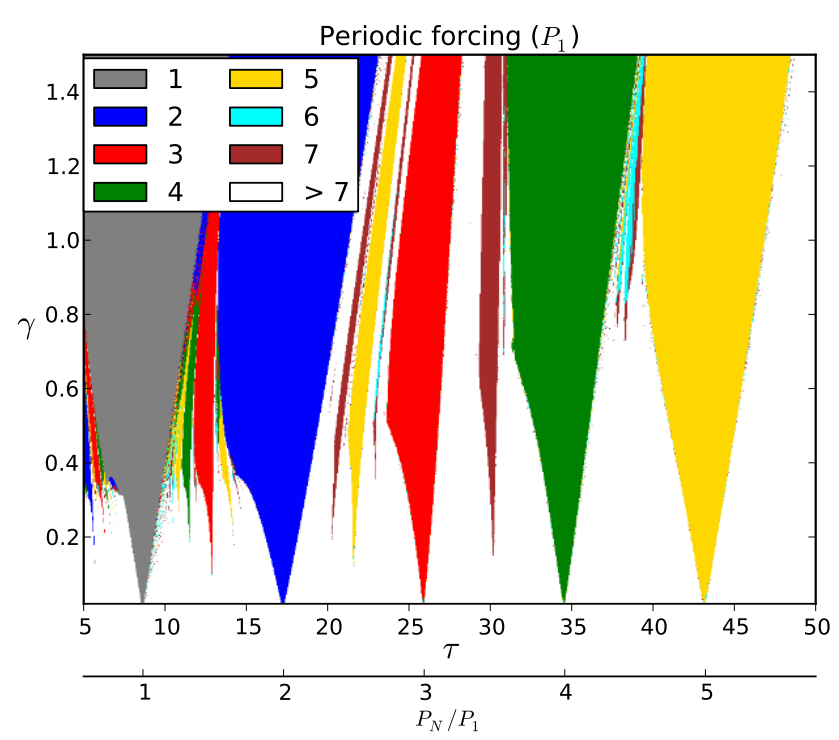

Fig. 6. Bifurcation diagram obtained by counting the number of points on the pullback section in the van der Pol oscillator $(\alpha=30$, $\beta=0.7)$ and $F(t)=\gamma \sin \left(2 \pi / P_{1} t+\phi_{P 1}\right)$. The two $x$ axes indicate $\tau$ and the ratio between the natural system period and the forcing, respectively. One observes the synchronisation regimes corresponding to $1: 1,1: 2,1: 3,1: 4$ and $1: 5$, respectively (gray, blue, red, green, yellow) and, intertwined, higher order synchronisations including $3: 2,5: 2,2: 3$ etc. White areas are weak or no synchronisation. Graph constructed using $t_{\text {back }}=-10 \mathrm{Ma}$ (see Fig. 11 and text for meaning and implications).

(1984), its occurrence in the van der Pol oscillator is discussed in Kapitaniak and Wojewoda (1990), and its relevance to climate dynamics was suggested by Sonechkin and Ivachtchenko (2001). In our specific example, the system is neither frequency-locked on $P_{1}$ nor on $O_{1}$, but it is synchronised in the generalised sense: the pullback section has two points. Finally, with $\tau=44 \mathrm{ka}$ there is frequency-locking on $O_{1}$ (regime $3: 1$ ) but not on $P_{1}$.

Clearly, the system underwent changes in synchronisation regimes as $\tau$ increased from 36 to $44 \mathrm{ka}$. Further insight may be had by considering the $\tau-\gamma$ plot (Fig. 8). The frequencylocking regime on $P_{1}$ lies in the relic of the $1: 4$ tongue visible in the periodic forcing case (Fig. 6). Frequency locking on $O_{1}$ belongs to the $1: 3$ tongue associated with $O_{1}$. The strange non-chaotic regime occurs where the tongues associated with these different forcing components merge.

The word bifurcation has been defined for nonautonomous dynamical systems (Rasmussen, 2000, Chap. 2). This is a complex subject and we will admit here the rather informal notion that there is a bifurcation when a local pullback attractor appears or ceases to exist (adapted from Def. 2.42 , in Rasmussen, 2000). With this definition, there is a bifurcation at least every time color changes in Fig. 8 (assuming $t_{\text {back }}$ is far enough in the past). 


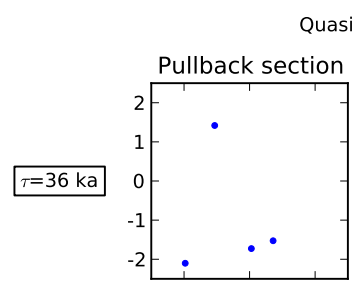

Quasi-periodic forcing $\left(P_{1}+E_{1}\right)$
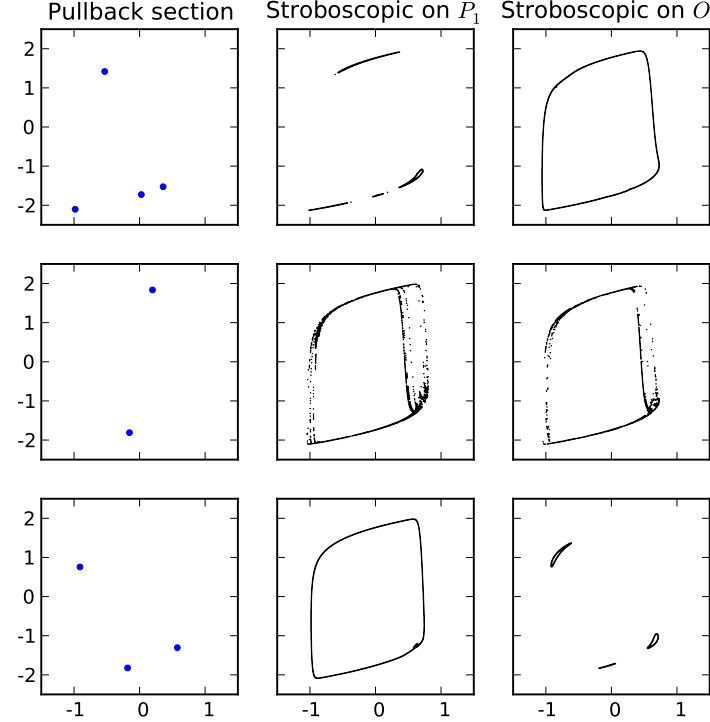

Fig. 7. Pullback (at $\left.t_{0}=0\right)$ and stroboscopic sections $\left(t=0+n P_{1}\right.$ and $t=0+n O_{1}$ ) obtained with the van der Pol oscillator with parameters $\alpha=30, \beta=0.7$, and forced by $F(t)=\gamma\left(\sin \left(2 \pi t / P_{1}+\right.\right.$ $\left.\left.\phi_{P 1}\right)+\sin \left(2 \pi t / O_{1}\right)+\phi_{O 1}\right), P_{1}=23.7 \mathrm{ka}$ and $O_{1}=41.0 \mathrm{ka}$ and $\gamma=0.6$, and three different values of $\tau$. The presence of dots on the pullback section indicates generalised synchronisation. Localised closed curves on the stroboscopic sections indicate frequency locking on the corresponding period (on $P_{1}$ with $\tau=36 \mathrm{ka}$ and $O_{1}$ with $\tau=44 \mathrm{ka}$ ), and complex geometries indicate the presence of a strange attractor $(\tau=41 \mathrm{ka})$.

Another view on the bifurcation structure may be obtained by plotting the $x$ and $y$ solutions of the system at $t_{0}=0$, initiated from a grid of initial conditions at $t_{\text {back }}=-5 \mathrm{Ma}$ (Million years), as a function of $\tau$, still with $\gamma=0.6$ (Fig. 9). This plot outlines a region of sensitive dependence on the input parameter, more specifically between $\tau=37$ and $42 \mathrm{ka}$.

These observations have two important consequences for our understanding of the phenomena illustrated in Fig. 1. To see this it is useful to refer to general considerations about autonomous dynamical systems. A bifurcation generally separates two distinct (technically: non-homeomorphic) attractors, which control the asymptotic dynamics of the system. As the bifurcation is being approached, the convergence to the attractor is slower, while the attractor that exists on the other side of the bifurcation may already take some temporary control on the transient dynamics of the system. This is, namely, one possible mechanism of excitable systems. One sometimes refers to "remnant" or "ghost attractors" to refer to these attractors that exist on the other side of the bifurcation and may take control on the dynamics of the system over significant time intervals (e.g. Nayfeh and Balachandran, 2004, p. 206).

The idea may be generalised to non-autonomous systems. Consider Fig. 10. The upper plot shows the two local
Quasi-periodic forcing $\left(P_{1}\right.$ and $\left.O_{1}\right)$

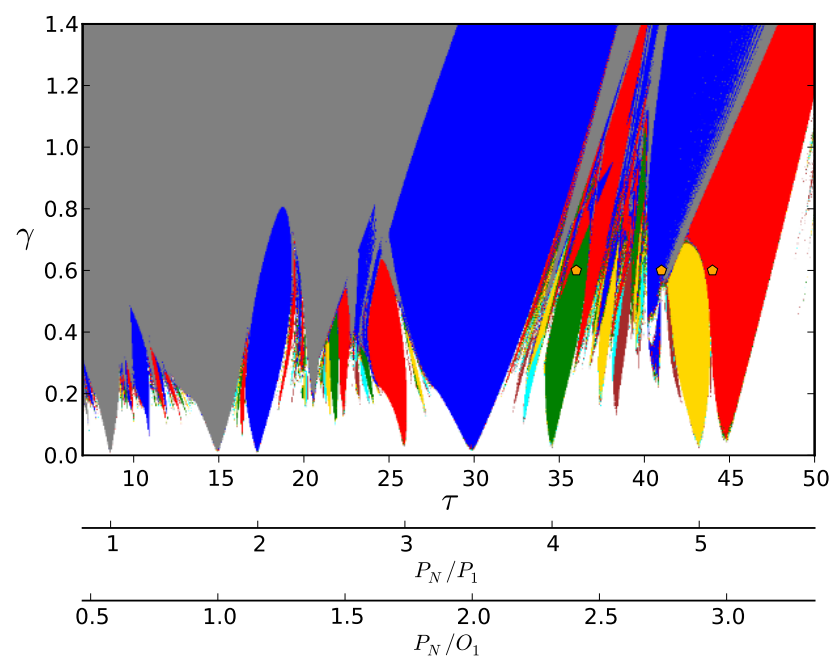

Fig. 8. As Fig. 6 but with a 2-period forcing: $F(t)=$ $\gamma\left(\sin \left(2 \pi / P_{1} t+\phi_{P 1}\right)+\sin \left(2 \pi / O_{1} t+\phi_{O 2}\right)\right)$ (see text for values). Tongues originating from frequency-locking on individual periods merge and give rise to strange non-chaotic attractors. Orange dots correspond to the cases shown in Fig. 7.

pullback attractors of the system obtained with $\tau=41 \mathrm{ka}$. The middle panel displays one local attractor obtained with $\tau=40$. The two $\tau=41$ attractors are reproduced with thin lines for comparison. Observe that this $\tau=40$ attractor is qualitatively similar to the $\tau=41$ attractors, and most of its time is spent on a path that is nearly undistinguishable from those obtained with $\tau=41$. However, on a portion of the time interval displayed it follows a sequence of osciliations that is distinct from those obtained with $\tau=41$. In fact, there are four pullback attractors at $\tau=40$.

Let us now consider a third scenario. Parameter $\tau=41$, but an additive stochastic term $\left(\sigma \frac{\mathrm{d} \omega}{\mathrm{d} t}, \sigma^{2}=0.25 \mathrm{ka}^{-1}\right.$, and $\omega$ symbolises a Wiener process) is added to the second system equation. This is thus a slightly noisy version of the original system. Shown here is one realisation of this stochastic equation, among the infinity of solutions that could be obtained with this system. Expectedly, the system spends large fractions of time near one or the other of the two pullback attractors. However, it also spends a significant time on a distinct path. Speculatively, this distinct path is under the influence of a "ghost" pullback attractor. As the bifurcation structure is complex and dense, as shown in Fig. 9, we expect a host of ghosts to lie around, ready to take control of the system over significant fractions of time, and this is what happens in this particular case.

To further support this hypothesis, consider a second experiment. Figure 11 displays the number of distinct solutions counted at time $t_{0}=0$, when the system is started from 121 distinct initial conditions at a time back $t_{\text {back }}$, as a function of 

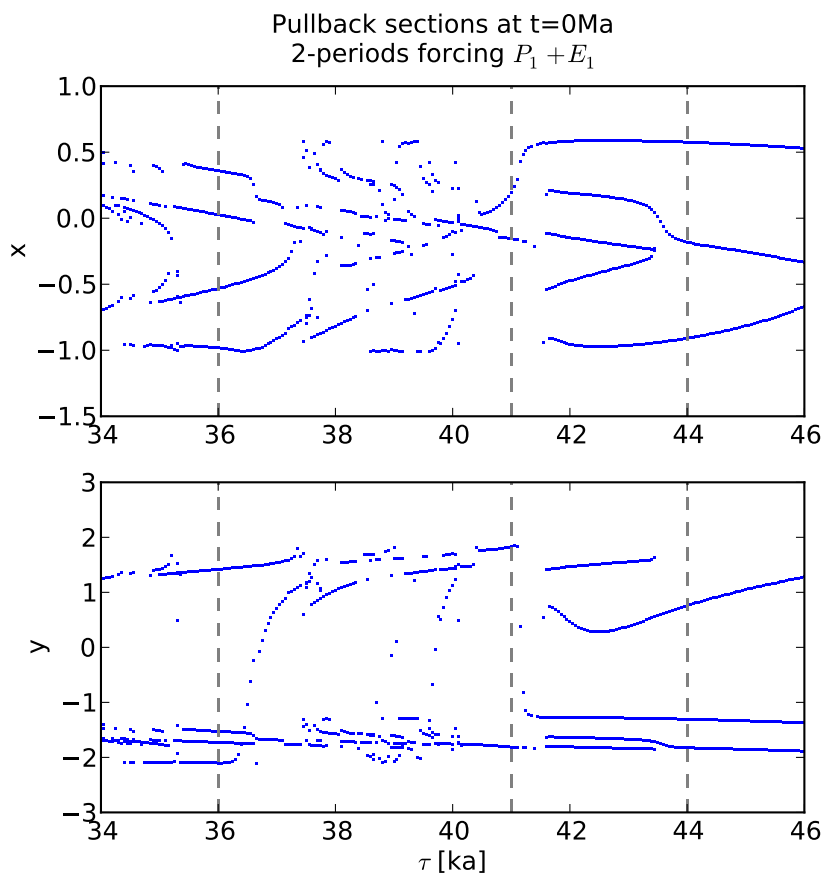

Fig. 9. Pullback solutions of the van der Pol oscillator forced by two periods (as in Figs. 7 and 8) as a function of $\tau$. Forcing amplitude is $\gamma=0.6$ and the other parameters are as on the previous figures. Vertical lines indicate the cases shown in Fig. 7.

$t_{\text {back. }}$. Surprisingly, one needs to go back to -30 Ma to identify the true pullback attractor. Obviously $30 \mathrm{Ma}$ is a very long time compared to the Pleistocene and so this solution is in practice no more relevant than the 4 or 8 solutions that can be identified by only starting the system back in time 1 or $2 \mathrm{Ma}$ ago. They may be interpreted as ghost pullback attractors, and following the preceding discussion they are likely to be visited by a system forced by large enough random external fluctuations.

\subsection{2 $P_{2}=22427 \mathrm{ka}$ and $P_{3}=18976 \mathrm{ka}$}

The two periods now being combined are the second and third components of precession, still according to Berger (1978). These two periods were selected for two reasons. The first one is that the addition of the two periodic signal produces an interference beating with period $123319 \mathrm{yr}$, not too far away from the usual $100 \mathrm{ka}$ cycle that characterises Late Pleistocene climatic cycles. Second, the period of the beating is not close to an integer number of the two original periods (this occurs, accidentally, when using $P_{1}$ and $P_{3}$ ). This was important to be able to clearly distinguish a synchronisation to the beating from a higher resonance harmonic to either forcing components.

It is known from astronomical theory that the periodicity of eccentricity is mechanically related to the beatings of the precession signal (Berger, 1978). The scientific question
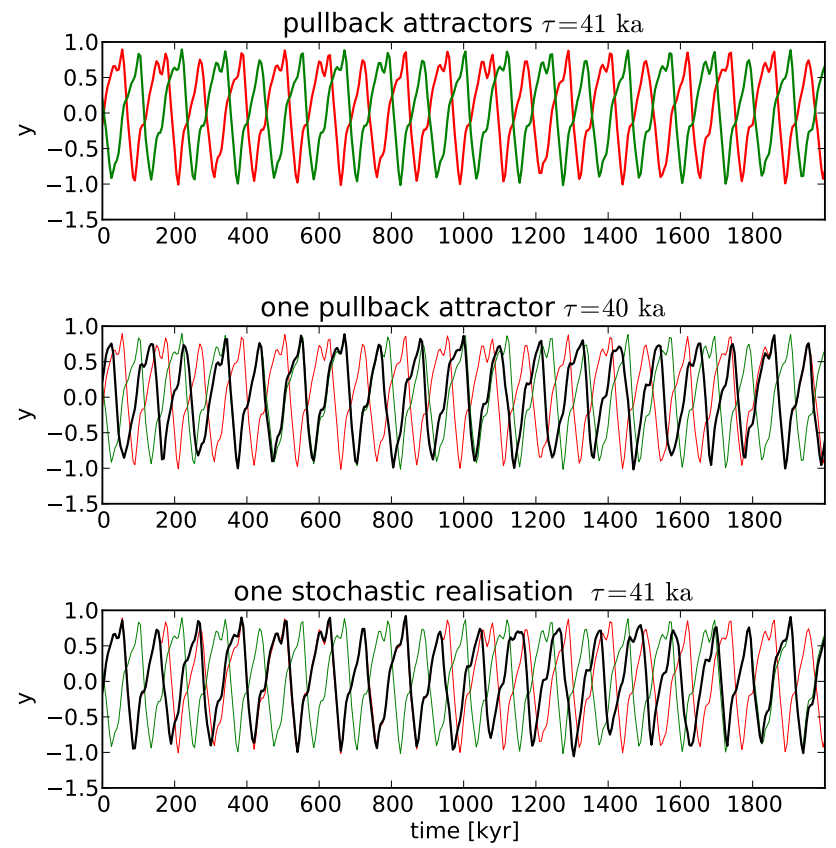

Fig. 10. (Top) pullback attractors of the forced van der Pol oscillator ( $\beta=0.7, \alpha=30, \gamma=0.6$ with 2-period forcing) as in Fig. 8, for $\tau=41 \mathrm{ka}$. They are reproduced on the graphs below (very thin lines), overlain by (middle) one pullback attractor with same parameters but $\tau=40 \mathrm{ka}$, and (bottom) one stochastic realisation of the stochastic van der Pol oscillator with $\tau=41 \mathrm{ka}$.

considered here is whether the correspondence between the period of ice age cycles and eccentricity is coincidental, or whether a phenomenon of synchronisation of climate on eccentricity developed.

To address this question we need a marker of synchronisation on the precession beating. The Rayleigh number has already been used to this end in palaeoclimate applications (Huybers and Wunsch, 2004; Lisiecki, 2010). Let $P_{\mathrm{b}}$ be the beating period, and $X_{i}$ the system state snapshot every $t=t_{0}+i P_{\mathrm{b}}$, the Rayleigh number $R$ is defined as $\left|\sum X_{i}-\bar{X}\right| / \sum\left|X_{i}-\bar{X}\right|$, where the overbar denotes an average. $R$ is strictly equal to 1 when the solution is synchronised with a periodic forcing of period $P_{\mathrm{b}}$, assuming no other source of fluctuations. As a reference, Lisiecki (2010) estimated 0.94 the Rayleigh number of a stacked benthic $\delta^{18} \mathrm{O}$ signal with respect to eccentricity over the last million years.

The bifurcation diagram showing the number of pullback solutions is displayed in Fig. 12. The frequency-locking tongues on $P_{2}$ and $P_{3}$ are easily identified at low forcing amplitude; as forcing amplitude increases the tongues merge and produce generalised synchronisation regimes. Regions of synchronisation on $P_{\mathrm{b}}$, identified as $R>0.95$, are hashed. They occur when the natural system period is close to $P_{\mathrm{b}}$ but narrow bands also appear near $T_{N}=P_{\mathrm{b}} / 2$. Observe also that these synchronisation regimes are generally not unique (several pullback attractors co-exist), and additional 


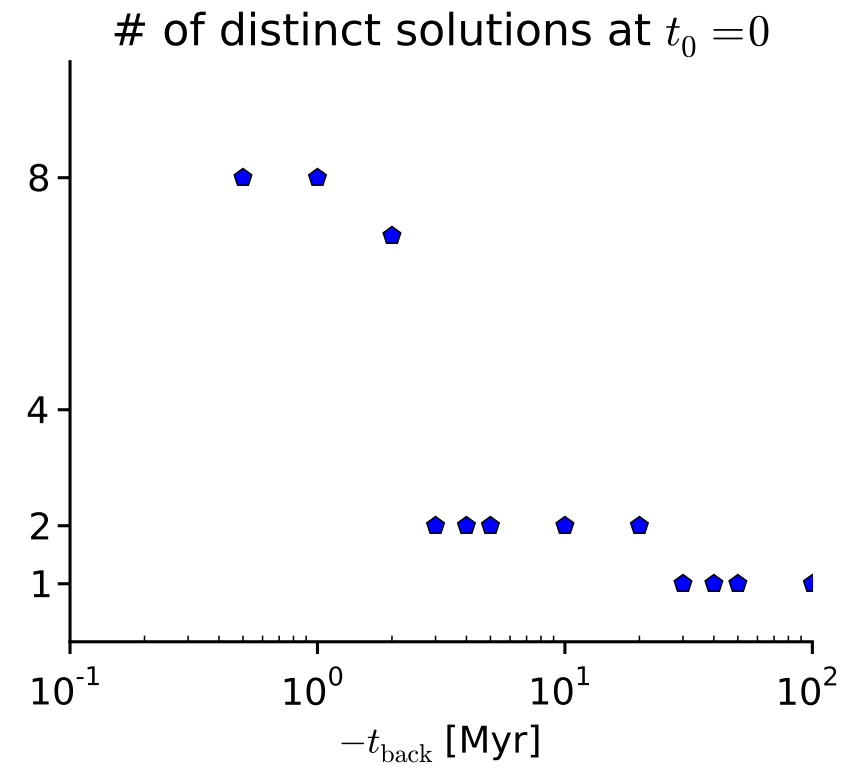

Fig. 11. Number of distinct solutions simulated with the van der Pol oscillator $(\beta=0.7, \alpha=30, \tau=41 \mathrm{ka}$ and $\gamma=0.6$ with 2-period forcing as in Fig. 8, as a function of the time $t_{\text {back }}$ at which 121 distinct initial conditions are considered. The actual stable pullback attracting set(s), in the rigorous mathematical sense, is (are) found for $t_{\text {back }} \rightarrow-\infty$.

sensitivity experiments show that convergence is quite slow. More specifically, the synchronisation diagram was computed here using $t_{\text {back }}=-10 \mathrm{Ma}$. With shorter backward time horizons, the number of remaining solutions identified in the beating-synchronisation regime often exceeds 6 and could not be seen on the graph, while the Rayleigh number was still beyond 0.95 . Hence, a high Rayleigh number is not necessarily a good indicator of reliable synchronisation.

\subsection{Full astronomical forcing}

The next step is to consider the full astronomical forcing, as the sum of standardised climatic precession $(\Pi)$ and the deviation of obliquity with respect to its standard value $(O)$ :

$F(t)=\gamma_{p} \Pi(t)+\gamma_{o} O(t)$

where

$$
\begin{gathered}
\Pi(t)=\sum_{i=1}^{N_{p}} a_{i} \sin \left(\omega_{p_{i}} t+\phi_{p_{i}}\right) / a_{1} \\
O=\sum_{i=1}^{N_{o}} b_{i} \cos \left(\omega_{o_{i}} t+\phi_{o_{i}}\right) / b_{1} .
\end{gathered}
$$

The various coefficients are taken from Berger (1978). We take $N_{p}=N_{o}=34$, so that the signal includes in total 68 harmonic components. With this choice the BER78 solution (Berger, 1978) is almost perfectly reproduced. BER78 is

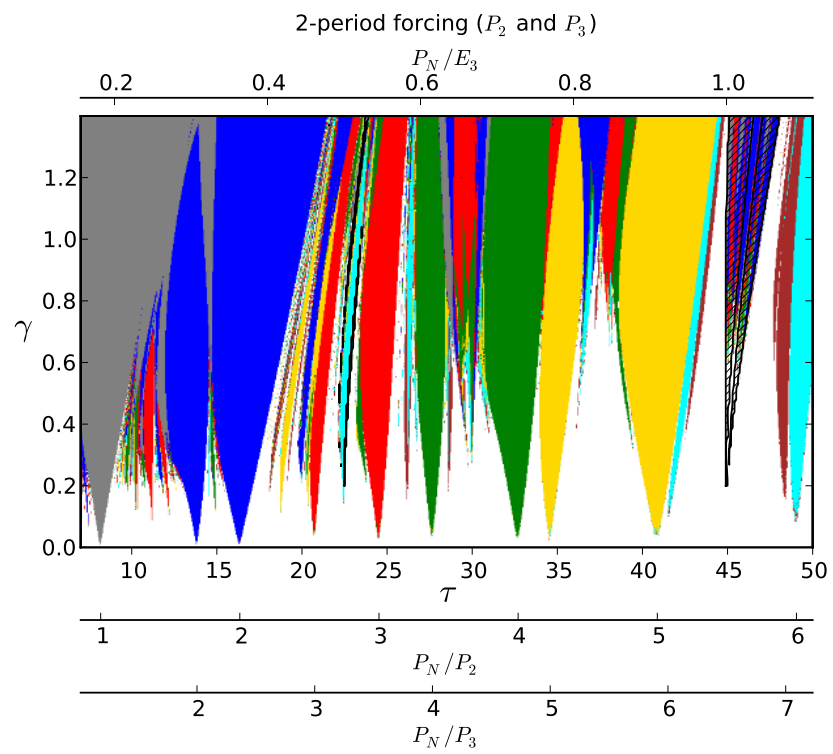

Fig. 12. As Fig. 8 but with: $F(t)=\gamma\left(\sin \left(2 \pi / P_{2} t+\phi_{P 2}\right)+\right.$ $\left.\sin \left(2 \pi / P_{3} t+\phi_{P 3}\right)\right)$. Hashes indicate regions of Rayleigh number $>0.95$ on the beating associated with $P_{2}$ and $P_{3}$, the period of which is called $E_{3}$ in the Berger (1978) nomenclature (third component of eccentricity).

still used in many palaeoclimate applications. Compared to a state-of-the-art solution such as La04 (Laskar et al., 2004), the error on amplitude is between 0 and $25 \%$, and the error on phase is generally much less than $20^{\circ}$.

The bifurcation diagram representing the number of pullback solutions as a function of forcing amplitude and $\tau$ is shown in Fig. 13. We have taken $\gamma=\gamma_{p}=\gamma_{o}$. One recognises the tongues originating from the individual components merging gradually into a complex pattern. The number of attractors settles to 1 as the amplitude of the forcing is further increased. Let us call this the 1-pullback attractor regime. We already know that synchronisation is generally not reliable in the region characterised by the complex and dense bifurcation region, where more than one attractor exist. The remaining problem is to characterise the reliability of synchronisation in the 1-pullback attractor regime.

The literature says little about systematic approaches to quantify the reliability of generalised synchronisation with quasi-periodic forcings. To develop further the ideas developed in Sect. 2.3.1, one can plot pullback solutions at a certain time $t$ as a function of one or several parameters. This is done in Fig. 14. Here $\gamma$ is kept constant $(=1.0)$ and $\tau$ is varied between 25 and 40 . There is a brief episode of a 2solution regime between 31 and $32 \mathrm{ka}$. Within the 1 -solution regime there is a number of abrupt transitions (at 26, 27, 34 and $37 \mathrm{ka}$ ).

Abrupt variations such as near $\tau=26,27,34$ and $37 \mathrm{ka}$ may represent discontinuities in the pullback attractor. In one case at least (at $\tau=34.46 \ldots$ ), the discontinuous character 


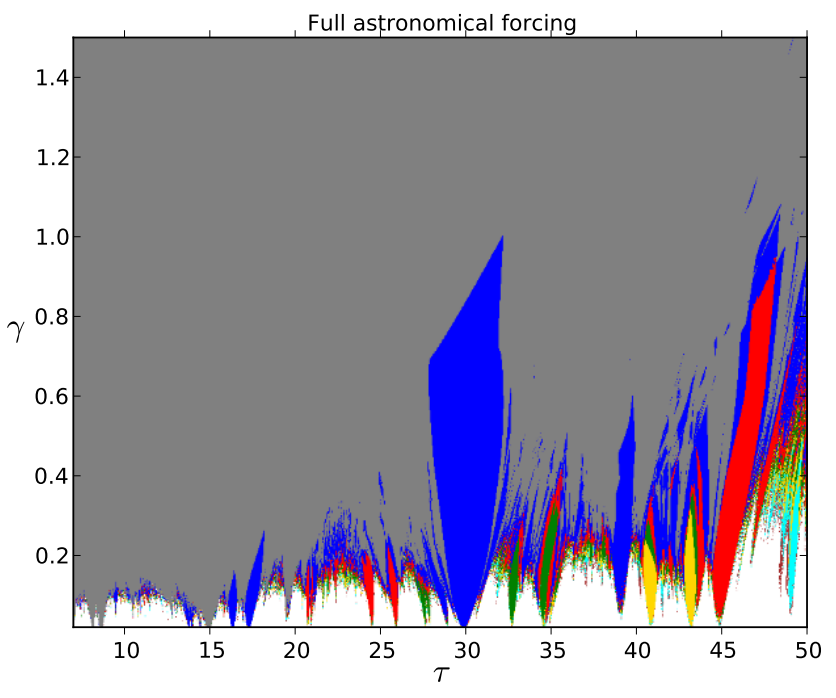

Fig. 13. As Fig. 8 but with the full astronomical forcing, made of 34 precession and 34 obliquity periods (Eq. 2).

could be verified up to machine precision. The abrupt variation at $\tau=37.59 \ldots$ appears numerically as a continuous variation of the attractor spread over an interval of $10^{-7} \mathrm{ka}^{2}$.

Further work is required to understand the origin of these discontinuities, and in particular to understand to what extent they relate to the strange nature of the attractor. The relevant aspect in the present context is that these discontinuities induce similar phenomena as those described in Sect. 2: the presence of ghost attractors and sensitive dependence to fluctuations. Hence, being in the 1-pullback attractor regime does not guarantee a reliable synchronisation on the astronomical forcing. One needs to be deep into that zone.

\section{Other models}

We now consider 6 previously published models. Mathematical details are given in the Appendix and the codes are available online at https://github.com/mcrucifix.

- SM90: this is a model with three ordinary differential equations representing the dynamics of ice volume, carbon dioxide concentration and deep-ocean temperature. The astronomical forcing is linearly introduced in the ice volume equation, under the form of insolation at $65^{\circ} \mathrm{N}$ on the day of summer solstice. Only the carbon dioxide equation is non-linear, and this nonlinearity induces the existence of a limit-cycle solution - spontaneous glaciation and deglaciation - in the corresponding autonomous system. The SM90 model is

\footnotetext{
${ }^{2}$ Note also that at least one discontinuity up to machine precision could also be identified in the 1-pullback attractor regime when $\alpha=$ 1 , which suggests that the feature is not conditioned by the slow-fast character of the system.
}
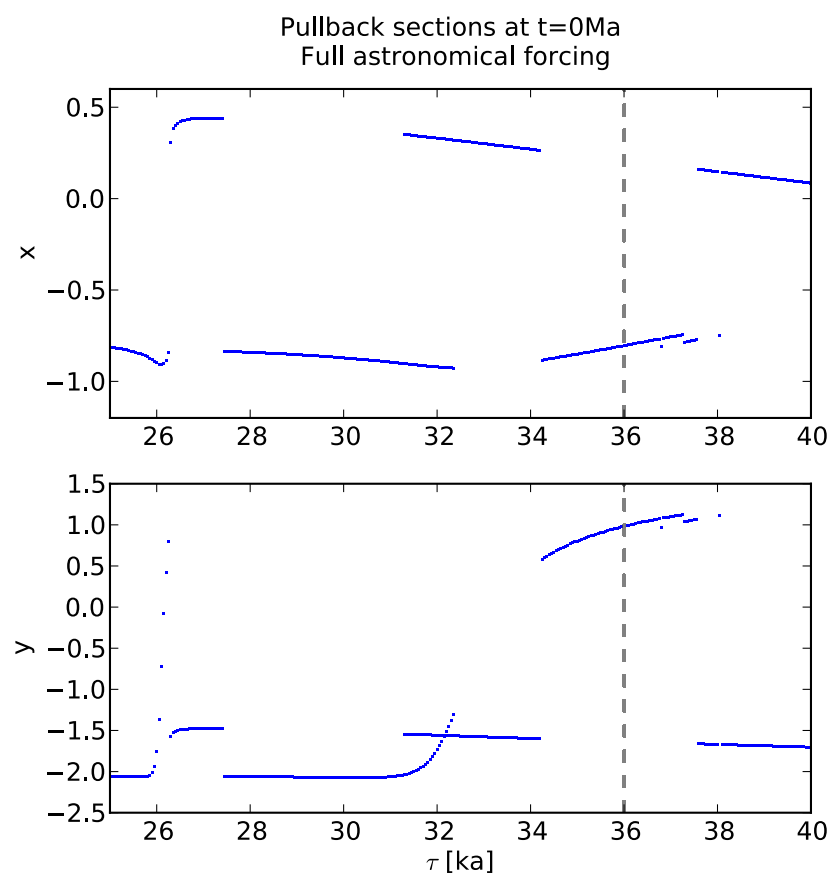

Fig. 14. As Fig. 9 but with the full astronomical forcing, with parameters as in Fig. 13 and $\gamma=1.0$.

thus a mathematical transcription of the hypothesis according to which the origin $100000 \mathrm{yr}$ cycle is to be found in the biological components of Earth's climate.

- SM91: this model is identical to SM90 except for a difference in the carbon cycle equation.

- PP04: the Paillard-Parrenin model (Paillard and Parrenin, 2004) is also a 3-differential-equation system, featuring Northern Hemisphere ice volume, Antarctic ice area and carbon dioxide concentration. The carbon dioxide equation includes one non-linear term associated to a switch on/off of the Southern Ocean ventilation. Astronomical forcing is injected linearly at three places in the model: in the ice-volume equation, in the carbon dioxide equation, and in the ocean ventilation parameterization. The autonomous version of the model also features a limit cycle. As in SM90 and SM91 the non-linearity introduced in the carbon cycle equation plays a key role but the bifurcation structure of this model differs from SM90 and SM91 (Crucifix, 2012).

- T06: the Tziperman et al. (2006) model is a mathematical idealisation of more complex versions previously published by Gildor and Tziperman (2000). T06 features the concept of sea-ice switch, according to which sea-ice growth in the Northern Hemisphere inhibits accumulation of snow over the ice sheets, and vice versa. Mathematically, T06 is presented as a hybrid model, 
which is the combination of a differential equation in which the astronomical forcing is introduced linearly as a summer insolation forcing term, and a discrete variable, which may be 0 or 1 to represent the absence or presence of sea ice in the Northern Hemisphere.

- I11: the Imbrie et al. (2011) was introduced by its authors as a "phase-space" model. It is a 2-D model, for which the equations were designed to distinguish an "ice accumulation phase" and an "abrupt deglaciation" phase, which is triggered when a threshold defined in the phase space is crossed. I11 was specifically tuned to reproduce the phase-space characteristics of the benthic oxygen isotopic dynamics. A particularity of this model is that the phasing and amplitude of the forcing depend on the level of glaciation.

- PP12: similar to Imbrie et al. (2011), the Parrenin and Paillard (2012) model distinguishes accumulation and deglaciation phases. Accumulation is a linear accumulation of insolation, without restoring force (hence similar to Eq. (1) of the van der Pol oscillator); deglaciation accumulates insolation forcing but a negative relaxation towards deglaciation is added. Contrary to Imbrie et al. (2011), the trigger function, which determines the regime change, is mainly a function of astronomical parameters. An ice volume term only appears in the function controlling the shift from "accumulation" to "deglaciation" regime.

The ice volumes (or, equivalently, glaciation index or sea level) simulated by each of these models are shown in Fig. 15. Shown here are estimates of the pullback attractors; more specifically, the trajectories obtained with an ensemble of initial conditions at $t_{\text {back }}=-20 \mathrm{Ma}$. In some cases the curves actually published (in particular in SM90) are not pullback attractors, but ghost trajectories in the sense illustrated in Fig. 11. In some cases (PP04 and PP12) the parameters had to be slightly adjusted to reproduce the published version satisfactorily. Details are given in Appendix.

Some of these models include as much as 14 adjustable parameters (e.g.: PP04) and a full dynamical investigation of each of them is beyond the scope of the study. Rather, we proceed as follows. Every model responds to a state equation, which may be written, in general (assuming a numerical implementation), as

$t_{i+1}=t_{i}+\delta t$

$x_{i+1}=x_{i}+\delta t f\left(x_{i}, F\left(t_{i}\right)\right)$,

where $t_{i}$ is the discretised time, $x_{i}$ the climate state (a 2-D or 3 -D vector) at $t_{i}$ and $F\left(t_{i}\right)$ is the astronomical forcing, which is specific to each model because the different authors made different choices about the respective weights and phases of precession and obliquity.
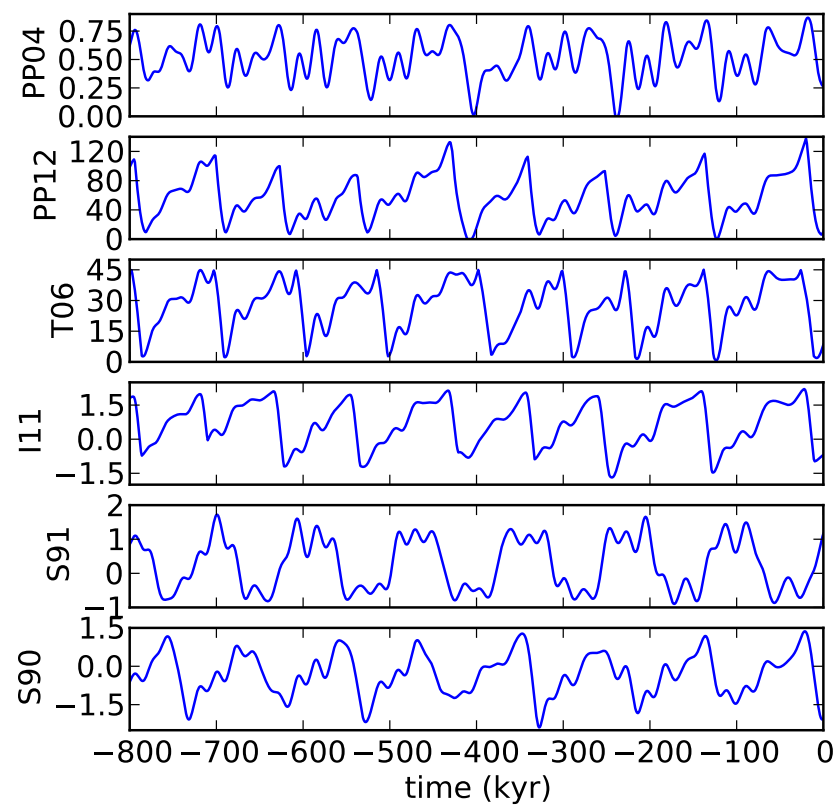

Fig. 15. Pullback attractors obtained for 6 models over the last $800 \mathrm{ka}$, forced by astronomical forcing with the parameters of the original publications. Shown is the model component representing ice volume. Units are arbitrary in all models, except in T06 (ice volume in $10^{15} \mathrm{~m}^{3}$ ) and PP12 (sea-level equivalent, in $\mathrm{m}$ ).

In all generality, the equation (or its numerical approximation) may be rewritten as follows, posing $\tau=1$ and $\gamma=1$ :

$$
\begin{aligned}
t_{i+1} & =t_{i}+\delta t \\
x_{i+1} & =x_{i}+\frac{1}{\tau} \delta t f\left(x_{i}, \gamma F\left(t_{i}\right)\right) .
\end{aligned}
$$

The parameters $\tau$ and $\gamma$ introduced this way have a similar meaning as in the van der Pol oscillator, since $\tau$ controls the characteristic response time of the model, while $\gamma$ controls the forcing amplitude.

Bifurcation diagrams, similar to Fig. 13 are then shown in Fig. 16. Remember that $\gamma=\tau=1$ corresponds to the model as originally published.

A first group of four models appears (SM90, SM91, T06 and PP04), on which one recognises a similar tonguesynchronisation structure as in the van der Pol oscillator. This was expected since these models are also oscillators with additive astronomical forcing. Depending on parameter choices synchronisation may be reliable or not. Synchronisation is clearly not reliable in the standard parameters used for SM90 and SM91. In T06 the standard parameters are not far away from the complex multi-pullback-attractor regime and this explains why transitions such as displayed in Fig. 1 could be obtained under small parameter changes (or, equivalently, with some noise, as discussed in the original Tziperman et al., 2006 study). The standard parameter choice of PP04 is further into the stability zone and, indeed, 

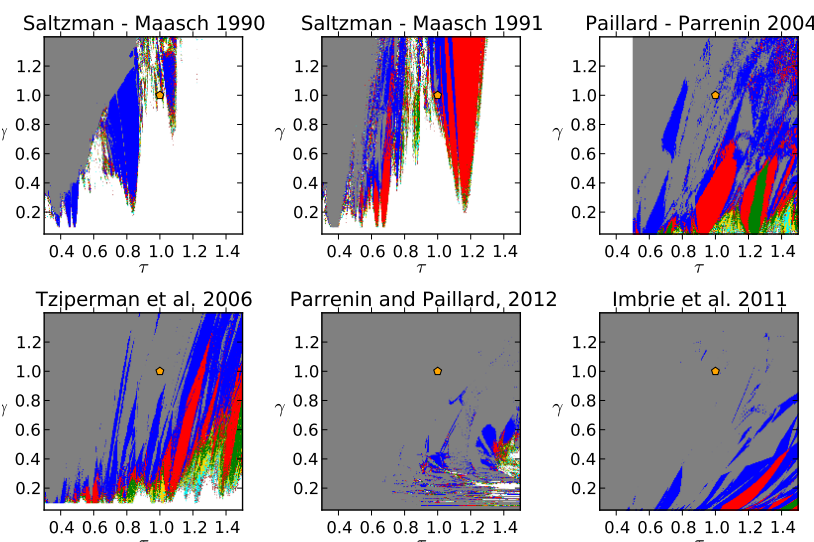

Fig. 16. As Fig. 13 but for 6 models previously published. Orange dots correspond to standard (published) parameters of these models.

experimenting with this model shows that instabilities such as displayed in Fig. 1 are harder to obtain.

I11, with standard parameters, is also fairly deep in the stable zone. One has to consider much smaller forcing values than published to recognise the synchronisation tongue structure that characterises oscillators (Fig. 17).

PP12 finally turns out to be the only case not showing a tongue-like structure. This may be surprising because this model also has limit-cycle dynamics (self-sustained oscillations in the absence of astronomical forcing). Some aspects of its design resemble the van der Pol oscillator. The role of the variable $y$ in the van der Pol oscillator is here played by the mode, which may either be $g$ (glaciation) or $d$ (deglaciation). Also similar to the van der Pol, the direct effect of the astronomical forcing on the ice volume ( $x$ in the van der Pol; $v$ in PP04) is additive. T06 has also similar characteristics. The distinctive feature of PP12 is that the transition between deglaciation and glaciation modes is controlled by the astronomical forcing and not by the system state, as in the other models. To assess the importance of this element of design we considered a hacked version of PP12, where the $d \rightarrow g$ occurs when for $v<v_{1}$ (cf. Appendix B6 for model details). In this case the tongue synchronisation structure is recovered, with standard parameters marginally in the reliable synchronisation regime (Fig. 17).

\section{Conclusions}

The present article is built around the paradigm of the "pacemaker", that is, the timing of ice ages arises as a combination of climate's internal dynamics with the variations of incoming solar radiation induced by the variations of our planet's orbit and obliquity. Mathematically, this implies that the models of ice ages tested here either present self-sustained oscillations in absence of astronomical forcing, or that the structure of the vector field is organised such as to excite
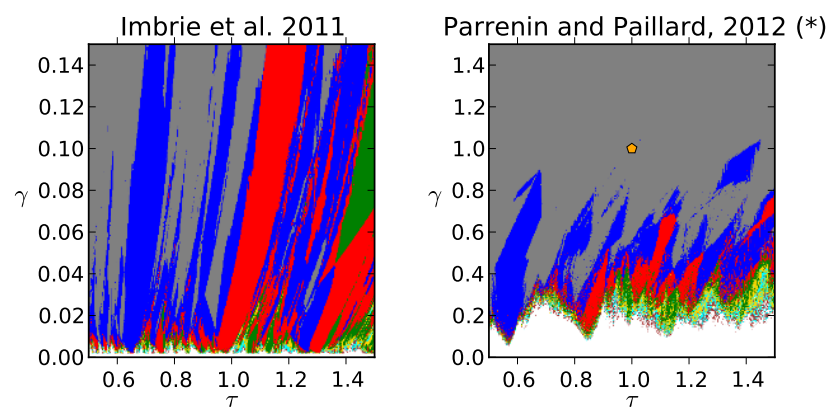

Fig. 17. As Fig. 16 for (left) the I1 1 model, but with a zoom on the $x$ axis, and (right) for a slightly modified version of the PP12 model, in which the transition between deglacial and glacial states is also controlled by ice volume, as opposed to the original PP12 model.

cyclic dynamics in the presence of a forcing. Once the forcing is present, the two scenarios will result in qualitatively similar dynamics (see Crucifix, 2012, for more discussion on this point).

In this study we paid attention to the dynamical aspects that may affect the stability of the ice age sequence and its predictability. First, the astronomical forcing has a rich harmonic structure. We showed that a system like the van der Pol oscillator is more likely to be synchronised on the astronomical forcing (as nature provides it) than on a periodic forcing, because the fraction of the parameter space corresponding to synchronisation is larger in the former case. A synchronised system is Lyapunov stable, so that at face value this would imply that the sequence of ice ages is stable. However, - this is the second point - even if the dynamical structure of the Pleistocene climate was correctly identified, there would be at least two sources of uncertainty : random fluctuations associated with the chaotic atmosphere and ocean and other statistically random forcings like volcanoes; and uncertainty on system parameters. In theory both types of uncertainties point to different mathematical concepts: path-wise stability to random fluctuations in the first case, and the structural stability in the second. In practice, however, lack of either form of stability will result in similar consequences: quantum skips of insolation cycles in the succession of ice ages. This was the lesson of Fig. 10.

It was shown here that, compared to periodic forcing, the richness of the harmonic structure of astronomical forcing favours situations of weak structural stability. The interpretation of the phenomenon relates directly to the theory of quasi-periodically forced dynamical systems, and the instability develops under relatively weak forcing. It is to be distinguished from the chaos known to develop at high forcing amplitude, and which has been implied in El-Niño theories Tziperman et al. (1994).

Out of the seven models tested here, we ignore which one best captures ice ages dynamics. The overwhelming complexity of the climate system does not allow us to securely 
select the most plausible model on the sole basis of our knowledge of physics, biology and chemistry. Consequently, while we have understood here how and why the sequence of ice ages could be unstable in spite of available evidence (astronomical spectral signature; Rayleigh number), estimating the stability of the sequence ice ages and quantifying our ability to predict ice ages is also a problem of statistical inference: calibrating and selecting stochastic dynamical systems based on both theory and observations, which are sparse and characterised by chronological uncertainties. A conclusive demonstration of our ability to reach this objective is still awaited.

\section{Appendix A}

\section{Insolation}

In the following models, the forcing is computed as a sum of precession $\left(\Pi=e \sin \varpi / a_{1}\right)$, co-precession $(\tilde{\Pi}=$ $\left.e \cos \varpi / a_{1}\right)$ and obliquity $\left(O=\left(\varepsilon-\varepsilon_{0}\right) / b_{1}\right)$ computed according to the Berger (1978) decomposition (Fig. 2, and Eqs. 3-4). More precisely, we use here these quantities scaled $(\bar{P}, \overline{\tilde{\Pi}}$ and $\bar{O})$ such as they have unit variance. All insolation quantities used in climate models may be approximated as a linear combination of $\bar{\Pi}, \bar{\Pi}$ and $\bar{O}$. For example:

- normalised summer solstice insolation at $65^{\circ} \mathrm{N}=$ $0.8949 \bar{\Pi}+0.4346 \bar{O}$

- normalised insolation at $60^{\circ} \mathrm{S}$ on the 21 February $=$ $-0.4942 \bar{\Pi}+0.8399 \overline{\tilde{\Pi}}+0.2262 \bar{O}$.

\section{Appendix B}

\section{Model definitions}

\section{B1 SM90 model}

$$
\begin{aligned}
& \frac{\mathrm{d} x}{\mathrm{~d} t}=-x-y-v z-u F(t) \\
& \frac{\mathrm{d} y}{\mathrm{~d} t}=-p z+r y+s z^{3}-w y z-z^{2} y \\
& \frac{\mathrm{d} z}{\mathrm{~d} t}=-q(x+z)
\end{aligned}
$$

$p=1.0, q=2.5, r=0.9, s=1.0, u=0.6, v=0.2$ and $w=$ 0.5 , and $F(t)$ is (here) insolation on the day of summer solstice, at $65^{\circ} \mathrm{N}$, normalised (results are qualitatively insensitive to the exact choice of insolation).

\section{B2 SM91 model}

$$
\begin{aligned}
& \frac{\mathrm{d} x}{\mathrm{~d} t}=-x-y-v z-u F(t) \\
& \frac{\mathrm{d} y}{\mathrm{~d} t}=-p z+r y-s y^{2}-y^{3} \\
& \frac{\mathrm{d} z}{\mathrm{~d} t}=-q(x+z)
\end{aligned}
$$

$p=1.0, q=2.5, r=1.3, s=0.6, u=0.6$ and $v=0.2$, and $F(t)$ is (here) insolation on the day of summer solstice, at $65^{\circ} \mathrm{N}$, normalised (results are qualitatively insensitive to the exact choice of insolation). One time unit is $10 \mathrm{ka}$.

\section{B3 PP04 model}

The three model variables are $V$ (Ice volume), $A$ (Antarctic Ice Area) and $C$ (Carbon dioxide concentration):

$$
\begin{aligned}
\frac{\mathrm{d} V}{\mathrm{~d} t} & =\frac{1}{\tau_{V}}\left(-x C-y F_{1}(t)+z-V\right) \\
\frac{\mathrm{d} A}{\mathrm{~d} t} & =\frac{1}{\tau_{A}}(V-A) \\
\frac{\mathrm{d} C}{\mathrm{~d} t} & =\frac{1}{\tau_{C}}\left(\alpha F_{1}(t)-\beta V+\gamma H+\delta-C\right),
\end{aligned}
$$

$\tau_{V}=15 \mathrm{ka}, \tau_{C}=5 \mathrm{ka}, \tau_{A}=12 \mathrm{ka}, x=1.3, y=0.4$ (was 0.5 in the original paper), $z=0.8, \alpha=0.15, \beta=0.5, \gamma=$ $0.5, \delta=0.4, a=0.4, b=0.7, c=0.01, d=0.27 ; H=1$ if $a V-b A+d-c F_{2}(t)<0$, and $H=0$ otherwise. $F_{1}(t)$ is the normalised, summer-solstice insolation at $65^{\circ} \mathrm{N}$, and $F_{2}(t)$ is insolation at $60^{\circ} \mathrm{S}$ on the 21 February (taken as $330^{\circ}$ of true solar longitude). Other quantities $(V, A, C)$ have arbitrary units.

\section{B4 T06 model}

The two model variables are $x$ (ice volume) and $y$ (sea-ice area). $x$ is expressed in units of $10^{15} \mathrm{~m}^{3}$.

$\frac{\mathrm{d} x}{\mathrm{~d} t}=\left(p_{0}-K x\right)\left(1-\alpha_{\mathrm{si}}\right)-\left(s+s_{\mathrm{m}} F(t)\right)$

The equation represents the net ice balance, as accumulation minus ablation, and $\alpha_{\mathrm{si}}$ is the sea-ice albedo. $\alpha_{\mathrm{si}}=$ 0.46y. $y$ switches from 0 to 1 when $x$ exceeds $45 \times 10^{6} \mathrm{~km}^{3}$, and switches from 1 to 0 when $x$ decreases below $3 \times$ $10^{6} \mathrm{~km}^{3}$. The parameters given by Tziperman et al. (2006) are: $p_{0}=0.23 \mathrm{~Sv}, K=0.7 /(40) \mathrm{ka}, s=0.23 \mathrm{~Sv}$ and $s_{\mathrm{m}}=$ $0.03 \mathrm{~Sv}$, where $1 \mathrm{~Sv}=10^{6} \mathrm{~m}^{3} \mathrm{~s}^{-1} . F(t)$ is the normalised, summer-solstice insolation at $65^{\circ} \mathrm{N}$. 


\section{B5 I11 model}

Define first:

$\phi=\frac{\pi}{180} \cdot \begin{cases}10-25 y & \text { where } y<0, \\ 10 & \text { elsewhere }\end{cases}$
$\theta= \begin{cases}0.135+0.07 y & \text { where } y<0, \\ 0.135 & \text { elsewhere }\end{cases}$

$a=0.07+0.015 y$

$h_{O}=(0.05-0.005 y) \bar{O}$

$h_{\Pi}=a \bar{\Pi} \sin \phi$

$h_{\tilde{\Pi}}=a \overline{\tilde{\Pi}} \cos \phi$

$F=h_{\Pi}+h_{\tilde{\Pi}}+h_{O}$

$d=-1+3 \frac{(F+0.28)}{0.51}$

With these definitions:

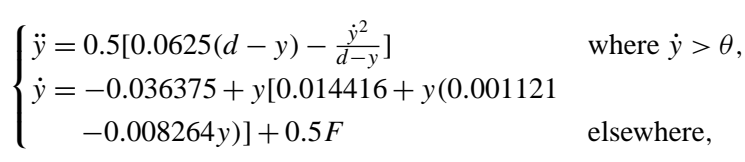

where $\dot{y}=\frac{\mathrm{d} y}{\mathrm{~d} t}$ and $\ddot{y}=\frac{\mathrm{d} \dot{y}}{\mathrm{~d} t}$. Note that the polynomial on the right-hand side of the equation for $\dot{y}$ is a continuous fit to the piece-wise function used in the original Imbrie et al. (2011) publication. Time units are here ka.

\section{B6 PP12 model}

This is a hybrid dynamical system, with ice volume $v$ (expressed in $m$ of equivalent sea level) and state, which may be $g$ (glaciation) or $d$ (deglaciation).

Define first

$f(x):= \begin{cases}x+\sqrt{4 a^{2}+x^{2}}-2 a & \text { where } x>0, \\ x & \text { elsewhere. }\end{cases}$

with $a=0.68034$. Then define the following quantities, standardised as follows:

$\Pi^{*}=(f(\bar{\Pi})-0.148) / 0.808$

$\tilde{\Pi}^{*}=(f(\overline{\tilde{\Pi}})-0.148) / 0.808$

the threshold $\theta=k_{\Pi} \bar{\Pi}+k_{\tilde{\Pi}} \overline{\tilde{\Pi}}+k_{O} \bar{O}$, and finally the following rule controlling the transition between state $g$ and $d$ :

$\left\{\begin{array}{ll}d \rightarrow g & \text { if } \theta<v_{1} \\ g \rightarrow d & \text { if } \theta+v<v_{0}\end{array}\right.$.
Ice volume $v$, expressed in sea-level equivalent, responds to the following equation:

$\frac{\mathrm{d} v}{\mathrm{~d} t}=-a_{\Pi} \Pi^{*}-a_{\tilde{\Pi}} \tilde{\Pi}^{*}-a_{O} \tilde{O}+ \begin{cases}a_{d}-v / \tau & \text { if state is } d \\ a_{g} & \text { if state is } g,\end{cases}$

with the following parameter values: $a_{\Pi}=1.456 \mathrm{mka}^{-1}$, $a_{\tilde{\Pi}}=0.387 \mathrm{mka}^{-1}, a_{O}=1.137 \mathrm{mka}^{-1}, a_{g}=0.978 \mathrm{mka}^{-1}$, $a_{d}=-0.747 \mathrm{mka}^{-1}, \quad \tau=0.834 \mathrm{ka}, \quad k_{\Pi}=14.635 \mathrm{~m}$, $k_{\tilde{\Pi}}=2.281 \mathrm{~m}, \quad k_{O}=23.5162 \mathrm{~m}, \quad v_{0}=122.918 \mathrm{~m} \quad$ and $v_{1}=3.1031 \mathrm{~m}$. This parameter set is the one originally presented by Parrenin and Paillard in Climate of the Past Discussion (which differs from the final version in Climate of the Past), except that $k_{O}$ is $18.5162 \mathrm{~m}$ in the original paper. This modification was needed to reproduce the exact sequence of terminations shown by the authors. Subtle details, such as the numerical scheme or the choice of the astronomical solution might explain the difference.

All codes and scripts are available from GitHub at https: //github.com/mcrucifix.

\section{Addendum}

The recently published article by Mitsui and Aihara (2013) further discusses this topic, by providing further support to the existence of strange non-chaotic attractors in van der Pol model as well as SM90, SM91 and PP04. They discuss also the question of sensitive dependence to fluctuations and their connection with the occurrence of strange non-chaotic attractors, with references to the works of Khovanov et al. (2000) in addition to those cited here.

Acknowledgements. Thanks are due to Peter Ditlevsen (Niels Bohr Institute, Copenhague), Frédéric Parrenin (Laboratoire de Glaciologie et de Géophysique, Grenoble), Bernard De Saedeleer, Ilya Ermakov and Guillaume Lenoir (Université catholique de Louvain) for comments on an earlier version of this manuscript. Two anonymous reviewers as well as Takahito Mitsui (FIRST, Aihara Innovative Mathematical Modelling Project, and University of Tokyo) provided useful comments that improved the Climate of the Past Discussion version. Thanks are also due to the numerous benevolent developers involved in the R, numpy and matplotlib projects, without which this research would have taken far more time. MC is research associate with the Belgian National Fund of Scientific Research. This research is a contribution to the ITOP project, ERC-StG grant 239604.

Edited by: J. Chappellaz 


\section{References}

Belogortsev, A. B.: Quasiperiodic resonance and bifurcations of tori in the weakly nonlinear duffing oscillator, Physica D, 59, 417429, doi:10.1016/0167-2789(92)90079-3, 1992.

Berger, A.: Support for the astronomical theory of climatic change, Nature, 268, 44-45, doi:10.1038/269044a0, 1977.

Berger, A. L.: Long-term variations of daily insolation and Quaternary climatic changes, J. Atmos. Sci., 35, 2362-2367, doi:10.1175/1520-0469(1978)035<2362:LTVODI>2.0.CO;2, 1978.

Crucifix, M.: Oscillators and relaxation phenomena in Pleistocene climate theory, Philos. T. R. Soc. A, 370, 1140-1165, doi:10.1098/rsta.2011.0315, 2012.

De Saedeleer, B., Crucifix, M., and Wieczorek, S.: Is the astronomical forcing a reliable and unique pacemaker for climate? A conceptual model study, Clim. Dynam., 40, 273-294, doi:10.1007/s00382-012-1316-1, 2013.

Feudel, U., Grebogi, C., and Ott, E.: Phase-locking in quasiperiodically forced systems, Phys. Rep., 290, 11-25, doi:10.1016/S0370-1573(97)00055-0, 1997.

Gildor, H. and Tziperman, E.: Sea ice as the glacial cycles climate switch: role of seasonal and orbital forcing, Paleoceanography, 15, 605-615, doi:10.1029/1999PA000461, 2000.

Grebogi, C., Ott, E., Pelikan, S., and Yorke, J. A.: Strange attractors that are not chaotic, Physica D:, 13, 261-268, 1984.

Glendinning, P., Feudel, U., Pikovsky, A. S., and Stark, J.: The structure of mode-locked regions in quasi-periodically forced circle maps, Physica D, 140, 227-243, doi:10.1016/S01672789(99)00235-3, 2000.

Guckenheimer, J., Hoffman, K., and Weckesser, W.: The forced van der Pol Equation I: the slow flow and its bifurcations, SIAM J. Appl. Dynam. Sys., 2, 1-35, doi:10.1137/S1111111102404738, 2003.

Hays, J. D., Imbrie, J., and Shackleton, N. J.: Variations in the Earth's orbit: pacemaker of ice ages, Science, 194, 1121-1132, doi:10.1126/science.194.4270.1121, 1976.

Huybers, P. and Wunsch, C.: A depth-derived Pleistocene age model: uncertainty estimates, sedimentation variability, and nonlinear climate change, Paleoceanography, 19, PA1028, doi:10.1029/2002PA000857, 2004.

Imbrie, J. and Imbrie, J. Z.: Modelling the climatic response to orbital variations, Science, 207, 943-953, doi:10.1126/science.207.4434.943, 1980.

Imbrie, J. Z., Imbrie-Moore, A., and Lisiecki, L. E.: A phase-space model for Pleistocene ice volume, Earth Planet. Sc. Lett., 307, 94-102, doi:10.1016/j.eps1.2011.04.018, 2011.

Kapitaniak, T. and Wojewoda, J.: Strange non-chaotic attractors of a quasi-periodically forced van der Pol's oscillator, J. Sound Vib., 138, 162-169, 1990.

Kapitaniak, T. and Wojewoda, J.: Attractors of Quasiperiodically Forced Systems, chap. 3, Strange Nonchaotic attractors, Wold Scientific publishing, 15-57, Singapore, 1993.

Khovanov, I. A., Khovanova, N. A., McClintock, P. V. E., and Anishchenko, V. S.: The effect of noise on strange nonchaotic attractors, Phys. Lett. A, 268, 315-322, doi:10.1016/S03759601(00)00183-3, 2000.

Laskar, J., Robutel, P., Joutel, F., Boudin, F., Gastineau, M., Correia, A. C. M., and Levrard, B.: A long-term numerical solution for the insolation quantities of the Earth, Astron. Astrophys., 428, 261-285, doi:10.1051/0004-6361:20041335, 2004.

Le Treut, H. and Ghil, M.: Orbital forcing, climatic interactions and glaciation cycles, J. Geophys. Res., 88, 5167-5190, doi:10.1029/JC088iC09p05167, 1983.

Lisiecki, L. E.: Links between eccentricity forcing and the 100000 -year glacial cycle, Nat. Geosci., 3, 349-352, doi:10.1038/ngeo828, 2010.

Lisiecki, L. E. and Raymo, M. E.: A Pliocene-Pleistocene stack of 57 globally distributed benthic $\delta^{18} \mathrm{O}$ records, Paleoceanography, 20, PA1003, doi:10.1029/2004PA001071, 2005.

Loutre, M. F.: Paramètres orbitaux et cycles diurne et saisonnier des insolations, $\mathrm{PhD}$ thesis, Université catholique de Louvain, Louvain-la-Neuve, Belgium, 1993.

Mettin, R., Parlitz, U., and Lauteborn, W.: Bifurcation structure of the driven Van der pol oscillator, Int. J. Bifurcat. Chaos, 3, 15291555, doi:10.1142/S0218127493001203, 1993.

Nayfeh, A. H. and Balachandran, B.: Applied Nonlinear Dynamics: Analytical, Computational, and Experimental Methods, Physics Textbook, Wiley-VCH Verlag, Weinheim, Germany, 2004.

Oerlemans, J.: Model experiments on the 100,000-yr glacial cycle, Nature, 287, 430-432, doi:10.1038/287430a0, 1980.

Mitsui, T. and Aihara, K.: Dynamics between order and chaos in conceptual models of glacial cycles, Clim. Dynam., online first, doi:10.1007/s00382-013-1793-x, 2013.

Paillard, D.: The timing of Pleistocene glaciations from a simple multiple-state climate model, Nature, 391, 378-381, doi:10.1038/34891, 1998.

Paillard, D.: Glacial Cycles: toward a New Paradigm, Rev. Geophys., 39, 325-346, doi:10.1029/2000RG000091, 2001.

Paillard, D. and Parrenin, F.: The Antarctic ice sheet and the triggering of deglaciations, Earth Planet. Sc. Lett., 227, 263-271, doi:10.1016/j.eps1.2004.08.023, 2004.

Saltzman, B. and Verbitsky, M. Y.: Multiple instabilities and modes of glacial rhythmicity in the plio-Pleistocene: a general theory of late Cenozoic climatic change, Clim. Dynam., 9, 1-15, doi:10.1007/BF00208010, 1993.

Parrenin, F. and Paillard, D.: Terminations VI and VIII $(\sim 530$ and $\sim 720 \mathrm{kyr}$ BP) tell us the importance of obliquity and precession in the triggering of deglaciations, Clim. Past, 8, 2031-2037, doi:10.5194/cp-8-2031-2012, 2012.

Pikovski, A., Rosenblum, M., and Kurths, J.: Synchronization: a Universal Concept in Nonlinear Sciences, Vol. 12, Cambridge Nonlinear Science Series, Cambridge University Press, New York, 2001.

Rasmussen, M.: Attractivity and Bifurcation for Nonautonomous Dynamical Systems, no. 1907 in Lecture Notes in Mathematics, Springer, Berlin Heidelberg, 2000.

Rial, J.: Earth's orbital eccentricity and the rhythm of the Pleistocene ice ages: the concealed pacemaker, Global Planet Change, 41, 81-93, doi:10.1016/j.gloplacha.2003.10.003, 2004.

Rial, J. A., Oh, J., and Reischmann, E.: Synchronization of the climate system to eccentricity forcing and the 100,000-year problem, Nat. Geosci., 6, 289-293,doi:10.1038/ngeo1756, 2013.

Romeiras, F. J. and Ott, E.: Strange nonchaotic attractors of the damped pendulum with quasiperiodic forcing, Phys. Rev. A, 35, 4404-4413, doi:10.1103/PhysRevA.35.4404, 1987. 
Saltzman, B. and Maasch, K. A.: A first-order global model of late Cenozoic climate, T. RSE Earth, 81, 315-325, doi:10.1017/S0263593300020824, 1990.

Saltzman, B. and Maasch, K. A.: A first-order global model of late Cenozoic climate - Part. II: Further analysis based on a simplification of the $\mathrm{CO}_{2}$ dynamics, Clim. Dynam., 5, 201-210, doi:10.1007/BF00210005, 1991.

Saltzman, B., Hansen, A. R., and Maasch, K. A.: The late Quaternary glaciations as the response of a 3-component feedbacksystem to Earth-orbital forcing, J. Atmos. Sci., 41, 3380-3389, 1984.

Schilder, F. and Peckham, B. B.: Computing Arnol'd tongue scenarios, J. Comput. Phys., 220, 932-951, doi:10.1016/j.jcp.2006.05.041, 2007.

Sonechkin, D. M. and Ivachtchenko, N. N.: On the Role of a quasiperiodic forcing in the interannual and interdecadal climate variations, CLIVAR exchanges, 6, 5-6, 2001.
Tziperman, E., Stone, L., Cane, M. A., and Jarosh, H.: El-Niño Chaos: Overlapping of resonances between the seasonal cycle and the pacific ocean-atmosphere oscillator, Science, 264, 7274, doi:10.1126/science.264.5155.72, 1994.

Tziperman, E., Raymo, M. E., Huybers, P., and Wunsch, C.: Consequences of pacing the Pleistocene $100 \mathrm{kyr}$ ice ages by nonlinear phase locking to Milankovitch forcing, Paleoceanography, 21, PA4206, doi:10.1029/2005PA001241, 2006.

Weertman, J.: Milankovitch solar radiation variations and ice age ice sheet sizes, Nature, 261, 17-20, 1976.

van der Pol, B.: On "relaxation-oscillations", The London, Edinburgh and Dublin, Philosophical Magazine Series 7, 2, 978-992, November, 1926.

Wiggins, S.: Chaos in the quasiperiodically forced duffing oscillator, Phys. Lett. A, 124, 138-142, doi:10.1016/03759601(87)90240-4, 1987. 